\title{
Forward and Backward Classical Conditioning of the Flexion Reflex in the Spinal Cat
}

\author{
Russell G. Durkovic* and Ernest N. Damianopoulos $\dagger$ \\ *Department of Physiology, SUNY-Upstate Medical Center, Syracuse, New York 13210, and †Department of \\ Psychology, Colgate University, Hamilton, New York 13346
}

\begin{abstract}
Effects of forward and backward conditioned-unconditioned stimulus (CS-US) intervals on classical conditioning of the flexion reflex were examined in a cat spinal preparation. Interstimulus intervals (ISIs) ranging from $+\mathbf{3 . 0}$ to $-\mathbf{3 . 0}$ sec were employed in 9 experimental groups and the results compared with those of an explicitly unpaired control group. Forward conditioning produced an asymmetrical, inverted U-shaped gradient relating magnitude of conditioning to ISI for both acquisition and extinction. The optimum ISI was 1.0 sec. Backward ISIs also produced excitatory conditioning, with optimal conditioning at -0.25 sec. Unlike forward conditioning, backward conditioning produced little sign of retention during extinction trials. The results, which parallel in several ways those of ISI effects in studies of intact animals, support the hypothesis that backward and forward conditioning may be fundamentally different phenomena, under the control of different neural processes.
\end{abstract}

As a simplified model for investigations of neural mechanisms of learning, the cat spinal preparation has been shown to exhibit the major behavioral features characterizing classically conditioned responses in intact animals (Durkovic, 1985b; Patterson, 1980). For example, spinal conditioning displays (1) conditioned increases in response to the conditioned stimulus (CS) over trials, reaching asymptotic levels (Durkovic, 1975, 1985a; Patterson et al., 1973); (2) sensitivity to conditioned (Misulis and Durkovic, 1984) and unconditioned (Durkovic and Light, 1975; Light and Durkovic, 1977) stimulus intensities; (3) dependence on contiguity of conditioned and unconditioned stimuli, as a number of different control procedures with unpaired CS and US (unconditioned stimulus) presentations fail to produce conditioning effects (Misulis and Durkovic, 1984; Patterson, 1980); and (4) retention of the conditioning effect (Durkovic, 1985a). The purpose of the present study was to examine the effects of interstimulus interval (ISI) on the development and maintenance of classically conditioned flexion reflex facilitation in this spinal preparation, and to compare the results to similar investigations in other preparations.

The ISI is one of the most important parameters affecting the outcome of classical conditioning procedures in intact animal studies (Gormezano et al., 1983). In general, when presentation of the CS precedes that of the US by a brief interval, optimum conditioning is observed. Studies of CS-US interval effects typically show an asymmetrical, inverted U-shaped gradient relating magnitude of conditioning to the ISI for "forward" conditioning (Hall, 1976; Jones, 1962; Schneiderman and Gormezano,

Received Dec. 9, 1985; revised Apr. 21, 1986; accepted Apr. 24, 1986

This research was supported by NSF Grants BNS 77-23845 and BNS 84-15917. The authors gratefully acknowledge the expert technical assistance of Stephen Onifer and the help in manuscript preparation of Terri McClelland.

Correspondence should be addressed to Dr. R. G. Durkovic at the above address. Copyright (C) 1986 Society for Neuroscience $0270-6474 / 86 / 102921-05 \$ 02.00 / 0$
1964). In contrast, successful "backward" conditioning (when US onset precedes CS onset) has been observed less frequently, and the very existence of backward conditioning has often been questioned (Cautela, 1965; Mackintosh, 1974). However, recent reviews of the subject argue for the acceptance of backward conditioning as a genuine excitatory conditioning phenomenon (Damianopoulos and Durkovic, 1986; Dostalek, 1976; Spetch et al., 1981). In the present study, use of CS-US intervals ranging from -3.0 to $+3.0 \mathrm{sec}$ allowed for tests of both forward conditioning and backward conditioning and for construction of an ISI function curve characterizing conditioning for this preparation. The data were further analyzed for contrasts between the responses induced by forward and backward conditioning paradigms in order to test the hypothesis that forward and backward conditioning arc products of different ncural processes.

Short notes dealing with preliminary results of this study have appeared previously (Durkovic 1980, 1985b).

\section{Materials and Methods}

One hundred adult male and female cats were used to form 1 control and 9 experimental treatment groups ( 10 animals/group). The animals were anesthetized with ether and, following tracheal cannulation, were provided with a mixture of $1-2 \%$ halothane in a $2: 1$ mixture of nitrous oxide and oxygen, using a Harvard constant-volume ventilator. Cannulation of the right carotid artery (to monitor blood pressure) and ligation of the left carotid artery followed. A laminectomy was performed and the spinal cord sectioned at $T-10$ with a thermal cautery. The halothane was discontinued and, after $1 \mathrm{~min}$, the animals were anemically decerebrated by clamping the vertebral arteries. At this point, the nitrous oxide-oxygen mixture was discontinued and the animal was artificially respirated with room air. An end-tidal $\mathrm{CO}_{2}$ level of 3-4\% and arterial blood pressure levels of $65-110 \mathrm{mmHg}$ were considered acceptable boundaries. Up to $10 \mathrm{ml} / \mathrm{kg}$ of a plasma expander (Rheomacrodex; $10 \%$ in normal saline) was administercd to help maintain blood pressure. Rectal temperature of $37^{\circ} \mathrm{C}$ was maintained by a heating pad and Ringer's lactated solution, at $10 \mathrm{ml} / \mathrm{hr}$, was administered through the forelimb vein throughout all subsequent procedures.

The animal's hindquarters were fixed in place with a clamp on the sacral vertebral processes and the left hind limb was rigidly fixed with a femur pin and toot clamp. The cutaneous superficial peroneal and saphenous nerves were isolated from surrounding tissue and placed over hook-shaped bipolar stimulating and recording electrodes. A mineral oil bath formed from surrounding loose skin housed the exposed nerves and electrodes. The distal tendon of the left tibialis anterior (TA) muscle was severed and attached to a transducer that converted mechanical tension to recording-pen deflection. Resting tension was 50-80 gm.

Following surgery, each of the 100 animals was allocated randomly to 1 of the 10 groups. Results from 11 additional animals either werc not obtained or had to be discarded because the physiological criteria for acceptability were not met. Of these, 5 animals did not meet the initial response criteria for rellex activity (see below). Three animals did not meet the mean arterial pressure requirement (these had arterial pressures below $58 \mathrm{mmHg}$ ). For 3 animals, the afferent nerve recordings were not stable over training (see below).

The CS was a $1.5 \mathrm{sec}$ train of $0.2 \mathrm{msec}$ electrical pulses at $10 / \mathrm{sec}$ applied to the exposed saphenous nerve. The US was a $0.5 \mathrm{sec}$ pulse 
Figure 1. ISI function curves for conditioning and extinction paradigms. Each point is the group mean $T A$ reflex change from preacquisition response to CS-alone trials ( \pm SEM). Conditioning, Mean change of $6 \mathrm{CS}$ alone "probe" trials during acquisition. Extinction, Mean change of 30 CS-alone trials following acquisition. Asterisks denote significant differences from $+30 \mathrm{sec}$ control group response changes.

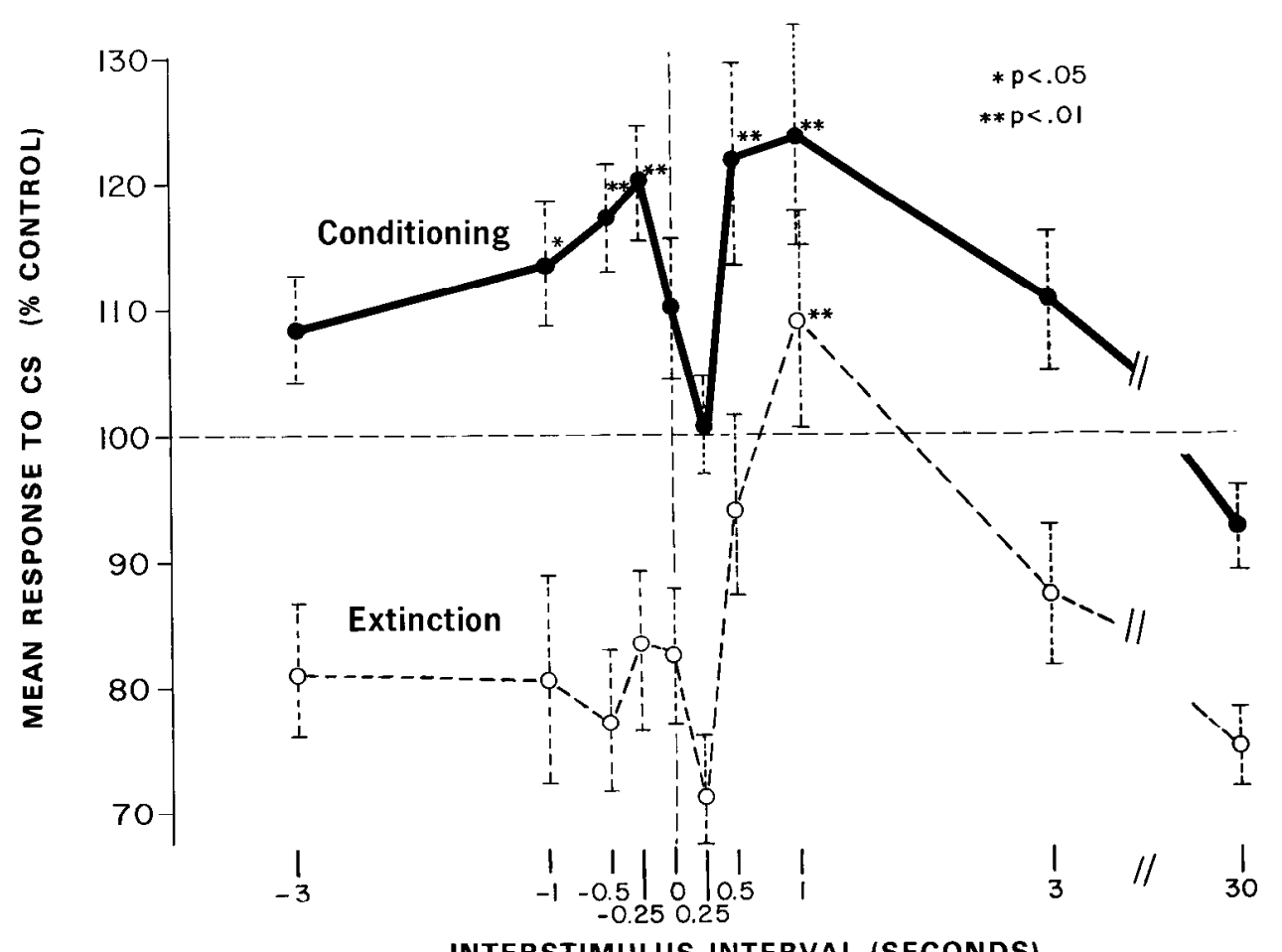

INTERSTIMULUS INTERVAL (SECONDS) train of $0.2 \mathrm{msec}$ pulses at $30 / \mathrm{sec}$ applied to the exposed superficial peroneal nerve. The intensities of the CS and US were set to activate A-alpha and A-delta cutaneous afferents supramaximally but were below $\mathrm{C}$-fiber thresholds. By monitoring the compound nerve action potentials from the saphenous and superficial peroneal cutaneous nerves, constant afferent nerve inputs were assured over the duration of each experiment (e.g., Durkovic, 1975, Fig. 2).

Treatment procedures were initiated after a minimum $2 \mathrm{hr}$ recovery and stabilization period following anemic decerebration. A test CS and test US with a $1 \mathrm{~min}$ ITI were administered to ensure that stimulating and recording instruments were functioning at appropriate intensity and gain levels, respcctively. Any subject failing to respond to the test CS with a reflex of at least $100 \mathrm{gm}$ in amplitude, and to the test US with a reflex of at least $350 \mathrm{gm}$ in amplitude was considered unacceptable, owing to possible nerve, muscle, or spinal cord dysfunction as a consequence of the surgical procedures (cf. Patterson, 1975, p. 87). Five min later, $5 \mathrm{CS}$-alone trials were administered at $1 \mathrm{~min}$ intervals to establish the $100 \%$ baseline reference level of responding. One minute later, CS presentations were given at $1 \mathrm{~min}$ constant ITI for all animals.

Subjects in each group received 30 CS-US presentations during acquisition. The CS-US interval varied, depending on group assignment, but was constant for all subjects within a group. The intervals were (CS onset to US onset) $0.25,0.5,1.0$, and 3.0 sec for the forward conditioning groups; $-0.25,-0.5,-1.0$, and $-3.0 \mathrm{sec}$ for the backward conditioning groups; and $0.0 \mathrm{sec}$ for the simultaneous conditioning group. Control treatment was unpaired CS-US presentations with a $30 \mathrm{sec}$ CS-US interval. Other types of unpaired (sensitization) control paradigms have been shown to lead to results similar to those induced using this procedure (e.g., Misulis and Durkovic, 1984, p. 89). Following acquisition, all animals received 30 additional CS-alone test trials at 1 min intervals to test for extinction.

The response measured was the maximum tension developed by the TA muscle during the first sec of the response to the CS. In order to monitor conditioned responding during acquisition, all animals were given CS-alone "probe" trials 1 min after the CS of CS-US trials 2, 6, $12,18,24$, and 30 . This was because the measure of the animal's response to the CS alone could not be obtained in groups in which the CS and US were presented closely together in time, because of the response to the unconditioned stimulus. These 6 probe responses were averaged and expressed as a percentage of the initial 5-trial CS-alone pretreatment baseline. The means of the $30 \mathrm{CS}$-alone extinction responses following acquisition were expressed in the same manner. Reflex responses to pretreatment CS-alone trials (mean of all animals $=461$ gm) did not vary among groups $(F(9,90)=0.379, p>0.1)$. The percentage changes from pretreatment baseline were analyzed by Dunnett's $t$ test, which is designed for examining multiple experimental group differences from a single control group (Edwards, 1968). A supplementary analysis of variance (Lundquist, 1953) was also conducted to obtain estimates of simple, interactive, and specific differences among certain forward, backward, and the control treatment groups.

\section{Results}

The ovcrall mean group responses for acquisition are presented in Figure 1. Forward conditioning produced flexion reflex facilitation, and a typical asymmetrical, inverted U-shaped curve of effect with optimum conditioning at $0.5-1.0 \mathrm{sec}$ was observed. Dunnett's $t$ test revealed significant differences $(p<0.01)$ compared to control group response levels for the 0.5 and $1.0 \mathrm{sec}$ groups, but no significant differences from control for the 0 , 0.25 , or $3.0 \mathrm{sec}$ ISI groups. Backward conditioning treatment also produced clear-cut excitatory conditioning effects, with optimal level of responding in the $-0.25 \mathrm{sec}$ group. Response levels of the $-0.25,-0.5$, and -1.0 sec ISI groups differed significantly from the control group response level $(p<0.05)$.

Mean response levels during extinction are also presented in Figure 1 , and it is here that differences between forward and backward conditioning results become apparent. Forward conditioning treatment produced an inverted U-shaped curve of effect in extinction, similar to that of acquisition. The maximum level of responding ( $+1.0 \mathrm{sec}$ group) was significantly greater than the control group response $(p<0.01)$. In contrast, responding in backward conditioning groups during extinction showed no evidence of retention.

Trial effects are shown in Figures 2-4. These figures show that a decline in responsivity to the CS occurred in the later stages of acquisition in subjects receiving control group treatment of unpaired CS-US presentations. In contrast, acquisition training in the paired groups resulted in rapid response increases early in training, although most groups paralleled control group behavior (but at higher levels) later in training. As expected 


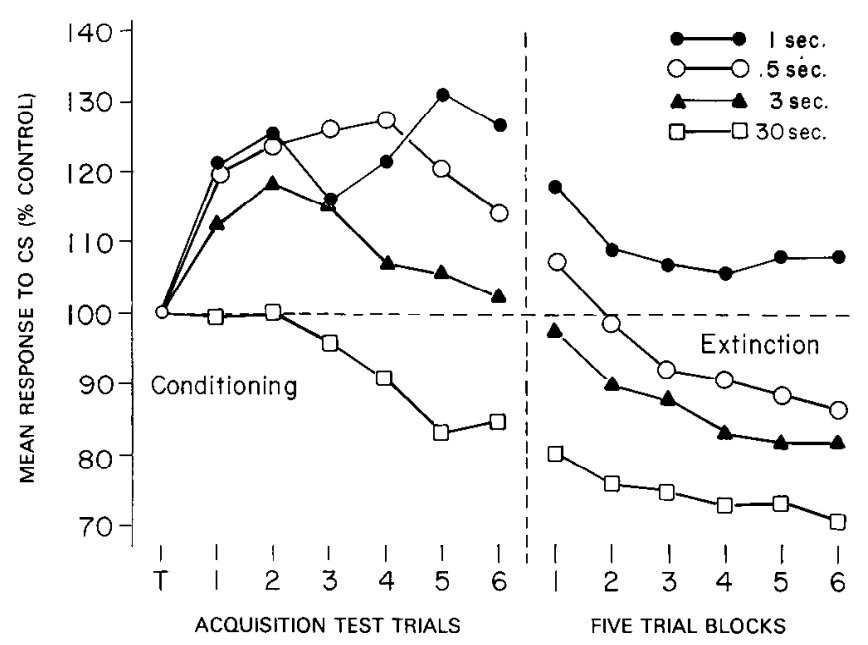

Figure 2. Trial effects during conditioning and extinction for the +3 , +1 , and $+0.5 \mathrm{sec}$ forward conditioning groups and the $+30 \mathrm{sec}$ unpaired control group. $T$, Mean control response to preacquisition CS-alone responses.

from the mean extinction results described above, CS-alone trials following acquisition produced rapid response decrements to control group levels in backward and simultaneous groups and in the $+0.25 \mathrm{sec}$ ISI group (Figs. 3 and 4). However, the forward conditioning groups presented in Figure 2 appeared to be more resistant to extinction effects.

To determine the reliability of these observations, as well as to make specific comparisons among backward conditioning (BC), forward conditioning (FC), and control group responding, a post hoc analysis of variance was carried out using the results of the best backward (ISI $=-0.25 \mathrm{sec}$ ), the best forward (ISI = $+1.0 \mathrm{sec}$ ), and the unpaired control treatment groups. Analysis of the 6 test trial data during acquisition indicated significant treatment $(F(2,27)=9.59, p<0.005)$, trials $(F(5,135)=2.63$, $p<0.025)$ and treatment $\times$ trials interaction $(F(10,135)=2.50$, $p<0.025)$ effects. Additionally, each test trial mean of the FC and BC groups exceeded those of the control group by more than the critical difference $(C D=19.72$ at $p<0.05)$. However, none of the differences in means for the respective test trials of the $\mathrm{BC}$ and $\mathrm{FC}$ groups exceeded this value. These data suggest that these selected FC and BC groups responded similarly during acquisition and that both showed facilitation compared to control group behavior.

A similar analysis of the 5-trial block extinction data indicated significant treatment $(F(2,27)=7.82, p<0.005)$, trial block $(F(5,135)=23.25, p<0.001)$ and treatment $\times$ trial block interaction $(F(10,135)=3.00, p<0.005)$ effects. In addition, the FC group means exceeded the control group means for each trial block and the $\mathrm{BC}$ means on all but the first trial block by more than the critical difference $(C D=19.14, p<0.05)$. In contrast, the differences between $\mathrm{BC}$ and control group trial block means were all less than this critical difference. These data reinforce the overall mean analysis of extinction, suggesting that a major difference exists between $\mathrm{FC}$ and $\mathrm{BC}$ group behavior, in that the latter shows little evidence of resistance to extinction procedures.

\section{Discussion}

The present results have implications for how well the spinal conditioning preparation models mammalian learning and memory behavior (and thus for the model's applicability to studying the neural mechanisms of such behavior); they are also relevant to a more specific understanding of backward and forward classical conditioning concepts.

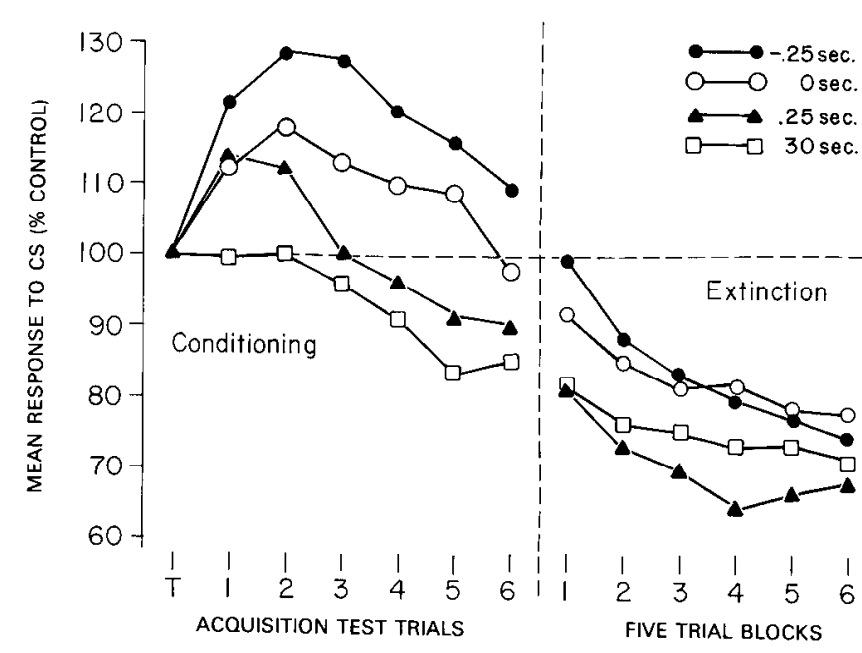

Figure 3. Trial effects during conditioning and extinction for the +0.25 , 0 , and $-0.25 \mathrm{sec}$ conditioning groups and the $+30 \mathrm{sec}$ unpaired control group.

Considering the model's characteristics first: A major finding of the present study is that CS-US interval effects in spinal conditioning replicate the asymmetrical, invertcd $U$-shaped gradients found for FC in intact animal studies (Gormezano et al., 1983). The observed effects were determined to be associative in nature, as paired trials resulted in increases in flexion reflex responding to the CS in many ISI groups, while control treatment produced no such effects. Furthermore, these results, together with earlier studies of CS and US intensity (Durkovic and Light, 1975; Light and Durkovic, 1977; and Misulis and Durkovic, 1984) and retention (Durkovic, 1985a), suggest that spinal conditioning is a valid simplified model of classical conditioning, as it replicates basic parameter effects found in intact animal studies.

Another major finding of the present study is the demonstration of BC according to the stated criteria (E. N. Damianopoulos and R. G. Durkovic, unpublished observations; Spetch et al., 1981). Treatment groups receiving backward intervals of -0.25 , -0.5 , and $-1.0 \mathrm{sec}$ responded at greater levels than control group subjects. Moreover, maximal level of facilitation attained appeared to be the same as that of FC. Interestingly, the simultaneous and backward conditioning results of the present

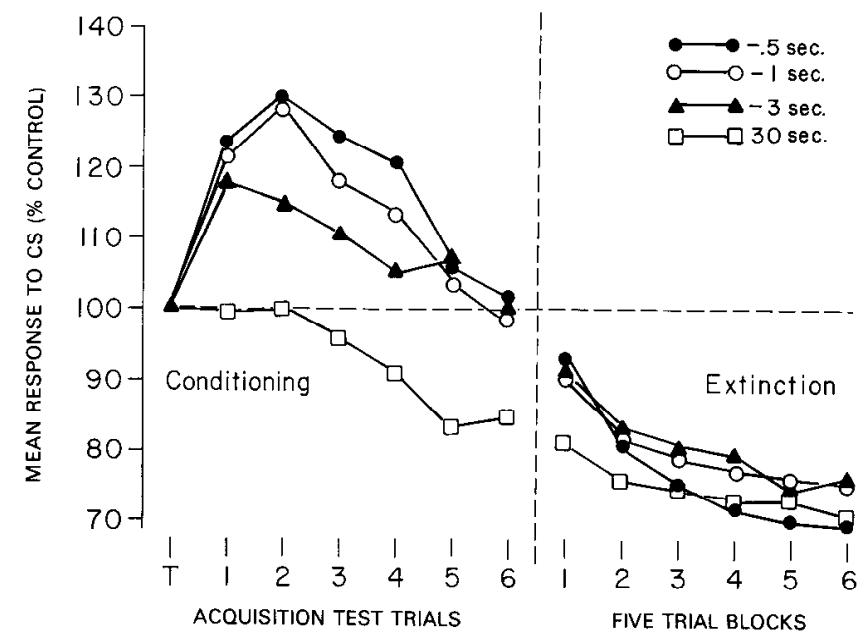

Figure 4. Trial effects during conditioning and extinction for the -0.5 , -1.0 , and $-3.0 \mathrm{sec}$ backward conditioning groups and the $+30 \mathrm{sec}$ unpaired control group. 


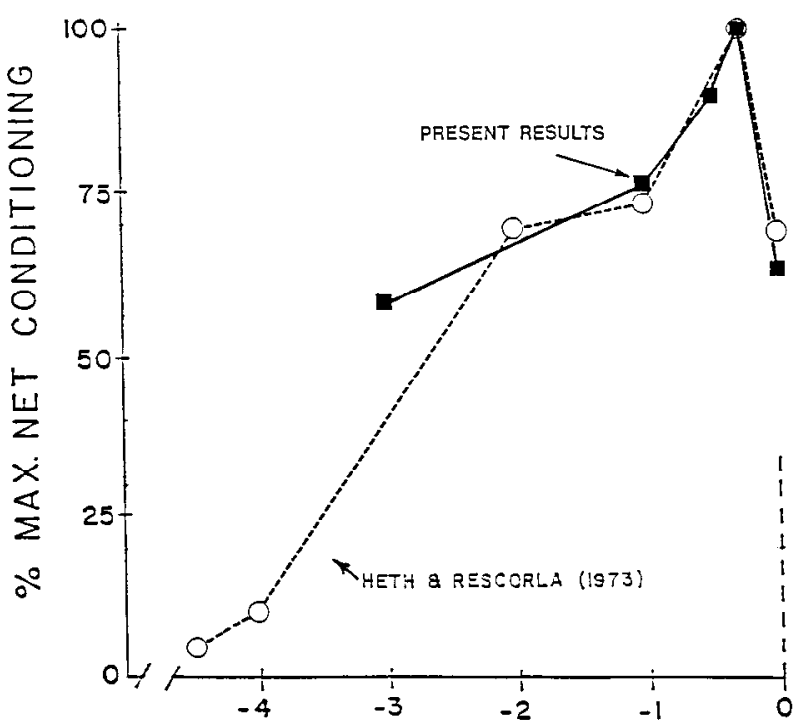

ISI (SECONDS)

Figure 5. ISI function curves for simultaneous and backward conditioning. Net conditioning is the numerical difference between experimental and control group treatment results (Light and Durkovic, 1977). The data from the 2 studies were normalized for comparison by expressing these values as percentages of the maximum net conditioning value. The data of the present results were obtained from the means of CS-alone trials during acquisition. The Heth and Rescorla (1973) curve is based on their averaged data from Experiments 1-3 (Fig. 4).

study bear a remarkable resemblance to the results of Heth and Rescorla (1973), who conducted an extensive investigation of rats as subjects in a bar-press suppression paradigm. Figure 5 shows the normalized data from the present study together with those of Heth and Rescorla (1973). As can be seen from this figure, simultaneous and backward ISIs produced closely approximating inverted V-shaped function curves in the 2 studies, with peak performance at an ISI of $-0.25 \mathrm{sec}$. Nonetheless, as with FC (Gormezano and Moore, 1969), backward ISI effects appear to be preparation-specific. For example, significant BC effects have been observed with ISI intervals as long as -5 to $-10 \mathrm{sec}$ in the studies of Matsumiya (1960) and Keith-Lucas and Guttman (1975). Furthermore, even in somewhat similar spinal conditioning studies of ISI effects, results different from those of the present study were observed (Patterson, 1975, 1980).

Patterson (1975) examined two BC groups (ISIs $=-0.25$ and $-0.1 \mathrm{sec}$ ) and one FC group (ISI $=+0.25 \mathrm{sec}$ ). While the forward paired group exhibited clear conditioned reflex facilitation, there was no statistical evidence of a change over conditioning trials in either backward group. Furthermore, Patterson (1980), in an examination of 9 groups with ISIs of $0.0-4.0 \mathrm{sec}$, reported a typical inverted $U$-shaped function curve, but with optimal conditioning with a $+0.25 \mathrm{sec}$ ISI.

While these findings clearly differ in major respects from those of the present study, experimental and preparation-specific differences that might account for the different results are readily identifiable: (1) Patterson used electrical stimulation of the superficial peroneal nerve as the CS, while in the present study the CS was stimulation of the saphenous nerve; (2) electrical stimulation of the skin of the ankle served as the US in Patterson's studies, while superficial peroneal nerve stimulation was used here; (3) Patterson's animals were paralyzed with a neuromuscular blocking agent, while animals in the present study were not paralyzed; (4) nerve activity induced in the deep peroneal nerve was recorded for measurement of conditioned reflex activity by Patterson, in contrast to the TA muscle tension recording in the present study; (5) Patterson's (1980) study employed a 0.5 -sec-duration US that terminated simultaneously with CS offset for all groups. Thus, the CS duration varied among groups, from 0.5 to $4.5 \mathrm{sec}$. In the present study, the same CS duration $(1.5 \mathrm{sec})$ was employed for all groups.

Some of the above factors descrve additional comment. For example, the use of neuromuscular blocking agents (3) has advantages in simplifying the neural model by preventing possible changes in reflex output caused by alterations in discharge from muscle afferents (e.g., Durkovic, 1976). However, recordings from the deep peroneal nerve (4) probably reflect activity from more than 1 muscle, and the outputs of individual flexor muscles have been shown to be influenced differently during spinal conditioning (e.g., Durkovic, 1983). Furthermore, deep peroneal nerve recordings may include a contribution from antidromic activation of muscle afferents (i.e., the dorsal root reflex; Brooks and Koizumi, 1956; Eccles et al., 1961), and therefore may reflect not only motor activity. Thus, the differences in ISI effects obtained from the 2 laboratories are likely a result of many major differences in experimental procedures.

Another finding of the present study (which has been documented in numerous intact animal studies) is that presentation of conditioned and unconditioned stimuli at intervals near simultaneity is relatively ineffective for the production of classically conditioned responses (see Fig. 1). This may be a result of teleologically derived processes designed to "protect" the nervous system from undergoing associatively produced changes by largely irrelevant, simultaneous sensory inputs that constantly occur in the environment. Such processes may function to supply brief inhibitory influences from one component of a sensory system onto other sensory and/or effector system components, and may be involved in other classes of observation, such as masking, blocking, overshadowing, and reflex inhibition (Estes, 1975; Ison and Reiter, 1980; Kling and Riggs, 1971). As a result of such processes, the nervous system components responsible for associative learning changes are less likely to be influenced by sensory inputs with near-simultaneous onsets. This does not, however, necessarily rule out the requirement for near-perfect synchronous activation of higher-order converging systems in order to obtain associative response alterations. For example, such a near-simultaneity requirement has been observed for the associative production of long-term potentiation of the crossed entorhinal cortical to dentate gyrus palhway (Levy and Steward, 1983).

Observations of excitatory $\mathrm{BC}$ are also relevant to general theories of classical conditioning, many of which require, by operational definition, that conditioning occur only when CS onset precedes US onset. However, in a review of the BC literature, Damianopoulos and Durkovic (1986) developed the concept that the existence of excitatory $\mathrm{BC}$ does not disconfirm such theories if, in fact, $\mathrm{BC}$ is a unique phenomenon compared to that of FC. The review cited examples in the literature in which BC and FC shared the basic indicators of learning: acquisition trends, retention, extinction, and ISI curves of effect. Nonetheless, BC appeared to differ from FC in CS intensity, US intensity, and trial density requirements. These differences were interpreted as being more consistent with an inference of a difference in neural mechanisms than with one of identity. Another characteristic in support of this conclusion has been observed in the present study: Rapid conditioned response decrement occurs in extinction in BC groups, in contrast to the resistance to extinction exhibited in FC. However, tests for retention, which generally require longer intervals between CS-alone trials (following conditioning) than are employed in this study (e.g., Durkovic, 1985a; Patterson, 1980), still need to be conducted for $\mathrm{BC}$ in this preparation. Finally, an even more important indicator of a difference between $\mathrm{BC}$ and $\mathrm{FC}$ mechanisms is the recent finding that the neural circuitries involved in $\mathrm{BC}$ and $\mathrm{FC}$ 
may be different (Durkovic and Onifer, 1985; S. Onifer and R. G. Durkovic, unpublished observations). While mechanisms underlying spinal conditioning have been proposed (Durkovic, $1985 \mathrm{~b}$ ), they have yet to be tested experimentally. Thus, further characterization of the mechanisms involved in $\mathrm{BC}$ and $\mathrm{FC}$, at this point, is not possible.

In summary, the present study reveals additional behavioral parallels between spinal conditioning and classically conditioned responding in intact animals in terms of both forward and backward ISI effects. Given the very real advantages of this model system for experimental analyses of the neural mechanisms underlying mammalian learning and memory (Durkovic, $1985 \mathrm{~b}$ ), the present results reemphasize the validity of this preparation for such studies. Additionally, the present spinal conditioning results, as well as others (Durkovic and Onifer, 1985), provide evidence in support of the hypothesis that forward and backward conditioning are unique phenomena under the control of different neural processes.

\section{References}

Brooks, C. M., and K. Koizumi (1956) Origin of the dorsal root reflex. J. Neurophysiol. 19: 61-74.

Cautela, J. R. (1965) The problem of backward conditioning. J. Psychol. 60: 135-144.

Dostalek, C. (1976) Backward conditioning in man and the criteria of conditioning. Activ. Nerv. (Suppl.). 18: 26-30.

Durkovic, R. G. (1975) Classical conditioning, sensitization and habituation in spinal cat. Physiol. Behav. 14: 297-304.

Durkovic, R. G. (1976) Aftereffects of static or dynamic fusimotor activation on primary afferent discharge. Exp. Neurol. 50: 99-112.

Durkovic, R. G. (1980) Interstimulus interval effects on classically conditioned flexion reflex facilitation in spinal cat. Soc. Neurosci. Abstr. 6: 191

Durkovic, R. G. (1983) Classical conditioning of the flexion reflex in spinal cat: Features of the reflex circuitry. Neurosci. Lett. 39: 155160.

Durkovic, R. G. (1985a) Retention of a classically conditioned reflex response in spinal cat. Behav. Neural Biol. 43: 12-20.

Durkovic, R. G. (1985b) The spinal cord: A simplified system for the study of neural mechanisms of mammalian learning and memory. In Development and Plasticity of the Mammalian Spinal Cord, M. E. Goldberger, A. Gorio, and M. Murray, eds., pp. 183-192 (Fidia Research Series, Vol. III), Liviana Press, Padova, Italy.

Durkovic, R. G., and A. R. Light (1975) Spinal conditioning: Unconditioned stimulus intensity requirement. Brain Res. 98: 364-368.

Durkovic, R. G., and S. Onifer (1985) Interstimulus interval effects on spinal conditioning using a low intensity CS. Soc. Neurosci. Abstr. 11: 1109.

Eccles, J. C., W. Kozak, and F. Magni (1961) Dorsal root reflexes of muscle group I afferent fibres. J. Physiol. (Lond.) 159: 128-146.

Edwards, A. L. (1968) Experimental Design in Psychological Research, p. 148, Holt, Rinehart and Winston, New York.
Estes, W. K. (ed.) (1975) Handbook of Learning and Cognitive Processes, Vol. 2: Conditioning and Behavior Theory, Lawrence Erlbaum, Hillsdale, NJ.

Gormezano, I., and J. W. Moore (1969) Classical conditioning. In Learning: Processes, M. H. Marx, ed., Macmillan, New York.

Gormezano, I., E. J. Kehoe, and B. S. Marshall (1983) Twenty years of classical conditioning research with the rabbit. Prog. Psychobiol. Physiol. Psychol. 10: 197-275.

Hall, J. F. (1976) Classical Conditioning and Instrumental Learning: A Contemporary Approach, Lippincott, Philadelphia.

Heth, C. D., and R. A. Rescorla (1973) Simultaneous and backward fear conditioning in the rat. J. Comp. Physiol. Psychol. 82: 434-443.

Ison, J. R., and L. A. Reiter (1980) Reflex inhibition and reflex strength. Physiol. Psychol. 8: 345-350.

Jones, J. E. (1962) Contiguity and reinforcement in relation to CSUCS intervals in classical aversive conditioning. Psychol. Rev. 69: 176-186.

Keith-Lucas, T., and N. Guttman (1975) Robust-single-trial delayed backward conditioning. J. Comp. Physiol. Psychol. 88: 468-476.

Kling, J. W., and L. A. Riggs (1971) Experimental Psychology, Holt, Rinehart and Winston, New York.

Levy, W. B., and O. Steward (1983) Temporal contiguity requirements for long-term associative potentiation/depression in the hippocampus. Neuroscience 8: 791-797.

Light, A. R., and R. G. Durkovic (1977) US intensity and blood pressure effects on classical conditioning and sensitization in spinal cat. Physiol. Psychol. 5: 81-88.

Lundquist, E. F. (1953) Design and Analysis of Experiments in Psychology and Education, pp. 266-273, Houghton Mifflin, New York.

Mackintosh, N. J. (1974) The Psychology of Animal Learning, pp. 5860 , Academic, New York.

Matsumiya, Y. (1960) The effects of US-intensity and CS-US pattern on conditioned emotional response. Jpn. Psychol. Res. 2: 35-42.

Misulis, K. E., and R. G. Durkovic (1984) Conditioned stimulus intensity: Role of cutaneous fiber size in classical conditioning of the flexion reflex in the spinal cat. Exp. Neurol. 86: 81-92.

Patterson, M. M. (1975) Effects of forward and backward classical conditioning procedures on a spinal cat hind-limb flexor nerve response. Physiol. Psychol. 3: 86-91.

Patterson, M. M. (1980) Mechanism of classical conditioning of spinal reflexes. In Neural Mechanisms of Goal-Directed Behavior and Learning, R. F. Thompson, L. Hicks, and V. B. Shvrykov, eds., pp. 263-272, Academic, New York.

Patterson, M. M., C. F. Cegavske, and R. F. Thompson (1973) Effects of a classical conditioning paradigm on hind-limb flexor nerve response in immobilized spinal cats. J. Comp. Physiol. Psychol. 84: 88-97.

Schneiderman, N., and I. Gormezano (1964) Conditioning of the nictitating membrane of the rabbit as a function of CS-US interval. J. Comp. Physiol. Psychol. 57: 188-195.

Spetch, M. L., D. M. Wilkic, and J. P. L. Pincl (1981) Backward conditioning: A re-evaluation of the empirical evidence. Psychol. Bull. 89: $163-175$. 